\title{
Treinamento funcional em homens com deficiência androgênica
}

\author{
Functional training in men with androgen deficiency
}

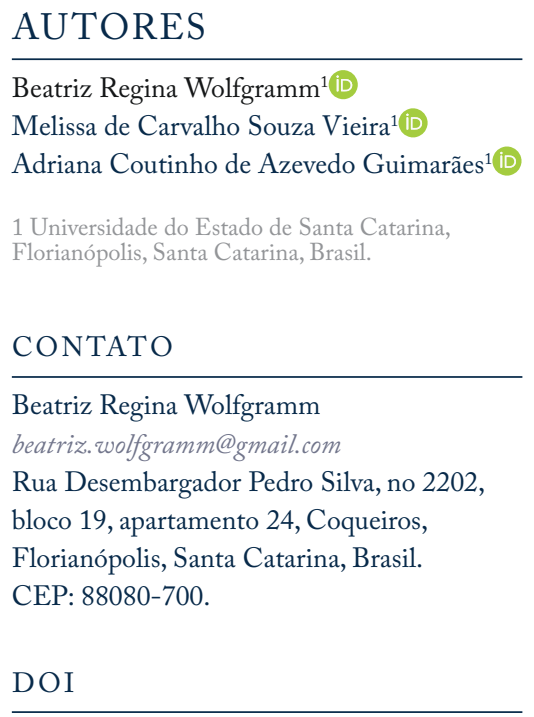

$10.12820 /$ rbafs. $23 \mathrm{e} 0040$

\begin{abstract}
RESUMO
Os sintomas da deficiência androgênica durante o envelhecimento masculino (DAEM) são evidenciados, a partir dos 40 anos, sendo que a atividade física (AF) pode atenuar esse processo. $\mathrm{O}$ objetivo desse estudo foi analisar a influência de um protocolo de treinamento funcional na $\mathrm{AF}$ habitual em homens com DAEM. Participaram 20 homens de 40 a 59 anos (média de idade de 49,63 $\pm 4,65$ anos), sendo 11 do grupo experimental e nove do grupo controle. Foram coletadas informações por meio de um questionário autoaplicável, dividido em três seções: características gerais e clínicas; AF - IPAQ (versão curta) e sintomas do envelhecimento masculino pela "Aging Male Symptoms Scale (AMS)”. Na análise dos dados, utilizou-se a estatística descritiva (média, desvio padrão e frequência simples) e inferencial (Exato de Fisher e Anova two way com medidas repetidas com teste de comparação de Sydak). O grupo experimental apresentou um aumento médio significativo no tempo de caminhada, em minutos por semana ( $265 \pm 53$ minutos; $\mathrm{p}=0,013)$, atividades moderadas $(138 \pm 21$ minutos; $\mathrm{p}=0,004)$, moderadas + vigorosas $(209 \pm 24$ minutos; $\mathrm{p}<0,001)$ e total $(474 \pm 48$ minutos; $\mathrm{p}<0,001)$ em comparação ao grupo controle. Um protocolo de treinamento funcional e a motivação da prática de AF foi eficaz para o aumento do tempo de prática de AF em homens com DAEM.
\end{abstract}

Palavras-chave: Atividade motora; Andropausa; Homens; Exercício.

\section{ABSTRACT}

The symptoms of androgen deficiency in the aging male (ADAM) affect men from the age of 40 , and in this context it is noted that the practice of physical activity (PA) can help in this process. Thus, the objective of this non-randomized clinical trial was to analyze the influence of a functional training protocol on habitual $P A$ in men with ADAM. Twenty men aged 40 to 59 years (mean age 49,63 4,65 years) 11 of the experimental group and 9 of the control group. Data were collected through a self-administered questionnaire divided into three sections: sociodemographic and clinical characteristics; Physical activity - IPAQ (short version) and symptoms of male aging by aging male symptoms scale (AMS); In which men responded in the pre and post-intervention, after being allocated into experimental and control groups. Statistical analysis was descriptive (mean, standard deviation and simple frequency) and inferential (Fisher exact, Anova two way tests with repeated measurements and Sydak comparison test). The experimental group presented a significant mean increase in walking time, in minutes per week (265 \pm 53 minutes, $p=0.013)$, moderate (138 \pm 21 minutes, $p=0.004)$, moderate + vigorous activities $(209 \pm 24$ minutes; $p<0.001)$ and total $(474$ \pm 48 minutes, $p<0.001$ ) compared to the control group. A functional training protocol and motivation for the practice of $P A$ was effective for increasing the practice of $A F$ in men with DAEM.

Keywords: Physical activity; Andropause; Men; Exercise.

\section{Introdução}

A pratica regular de atividade física $(\mathrm{AF})$ quando realizada de acordo com as recomendações ( $\geq 150$ minutos/semana) pode atuar na prevenção de diferentes doenças na população em geral ${ }^{1,2}$. Está amplamente documento na literatura que homens são fisicamente mais ativos do que mulheres ${ }^{3-4}$. Entretanto, na meia idade, há maior incidência de doenças crônicas nos homens ${ }^{5}$ e essas doenças estão associadas a preferência por atividades físicas mais leves ${ }^{6}$.

Além da redução da intensidade das $\mathrm{AF}$ práticas, têm sido observadas alterações morfológicas, psicoló- gicas e físicas e declínio gradual dos níveis de testosterona na população masculina com o passar da idade ${ }^{7-9}$. Tem sido observado que a partir dos 40 anos de idade são observados sintomas de deficiência androgênica do envelhecimento masculino $(\mathrm{DAEM})^{10}$. É possível que a presença destes sintomas tenha uma relação direta com o nível de $\mathrm{AF}$, tendo em vista que homens com maior prevalência e intensidade de sintomas, em geral, são os indivíduos insuficientemente ativos ${ }^{11}$.

Sendo assim, a manutenção dos níveis de prática de AF nesse período da vida é uma forma de melhorar 
o estado de saúde e os sintomas da DAEM em homens. Evidencia-se que para a melhora da saúde da população masculina é pertinente combinar exercícios aeróbios com exercícios resistidos, de intensidade moderada à vigorosa ${ }^{12-13}$.

Nesse sentido, o treinamento funcional combina os dois tipos de exercícios recomendados (exercícios aeróbios e resistidos) como uma opção para tratamento, manutenção e recuperação da saúde ${ }^{14}$, tendo em vista os seus efeitos positivos na saúde ${ }^{15-17}$ É possível que o treinamento funcional possa ser capaz de melhorar os níveis de força muscular, diminuir dores articulares e musculares, desânimo e irritabilidade, além de ser possível atuar como um incentivo para a prática e manutenção da AF atual nessa fase da vida. Sendo de extrema importância incorporar intervenções que contribuam para uma vida mais ativa de forma eficaz em adultos com DAEM. Entretanto, até o momento, ainda não se tem conhecimento de evidências sobre os seus benefícios nos aspectos somáticos, psicológicos e sexuais de homens com DAEM e como forma de manutenção dos níveis de AF dessa população. Assim, o estudo teve como objetivo investigar a influência de um protocolo de treinamento funcional na prática de $\mathrm{AF}$ habitual em homens com deficiência androgênica do envelhecimento masculino.

\section{Métodos}

Trata-se de um ensaio clínico não randomizado, aprovado pelo Comitê de Ética em Pesquisa em Seres Humanos da Universidade do Estado de Santa Catarina, sob o protocolo n.1.801.237 e no Registro Brasileiro de Ensaios Clínicos - REBEC: RBR-7x3tkf.

Participaram do estudo 20 adultos do sexo masculino, 11 alocados no grupo experimental (GE) e nove no grupo controle (GC), residentes em Florianópolis e São José, Santa Catarina, selecionados por meio de divulgação na mídia (rádio, jornal local, site da universidade e telefone) e em instituições de serviço de Educação, Saúde e Justiça.

Os interessados foram avaliados primeiramente pela Escala dos Sintomas do Envelhecimento Masculino (AMS), versão para o português ${ }^{18}$. Os adultos que apresentaram os sintomas clínicos da DAEM foram considerados elegíveis. Os que optaram por participar do treinamento foram alocados no $\mathrm{GE}$ e aqueles que não tiveram disponibilidade e/ou interesse para frequentar a intervenção, mas se interessaram em participar do estudo, fizeram parte do GC.
Os critérios de exclusão adotados foram: homens que possuíam histórico de doenças cardiovasculares, musculoesqueléticas, neurológicas, câncer de próstata; que tinham realizado treinamento funcional nos três meses anteriores à coleta de dados; que não atenderam ao contato telefônico por três vezes e; os que não alcançaram 75\% de frequência nas aulas.

O processo de seleção dos participantes e execução das etapas do estudo encontram-se na Figura 1.

Após a explicação dos objetivos do estudo, os participantes selecionados para o GC assinaram o termo de consentimento livre e esclarecido, e posteriormente realizaram as avaliações antes e após o período de 12 semana de intervenção. Ainda, receberam quinzenalmente, por meio de cartilhas informativas, diretrizes para a prática de exercícios físicos, como utilização de vestimenta adequada e hidratação. A coletada de dados da linha de base foi realizada em julho, seguindo o mesmo protocolo para os participantes do GC e GE.

A intervenção foi realizada no período de 22 de agosto a 30 de novembro de 2016, e consistiu de aulas de treinamento funcional, duas vezes por semana, por 60 minutos, durante 12 semanas. Além das duas sessões semanais de treinamento funcional, foi recomendado aos participantes do GE que os mesmos realizassem outras atividades físicas, como caminhada, bicicleta, e/ou corrida, afora o deslocamento ativo - ir de bicicleta ou a pé para o trabalho, subir escadas ao invés de usar o elevador, lavar o carro, durante os demais dias da semana, com objetivo de aumentar o nível de AF (ser ativo o maior tempo possível). No GE, após a intervenção, todos foram orientados a continuar a prática do treinamento funcional, tendo em vista que já estavam praticando a modalidade há três meses. O GC recebeu panfletos informativos sobre AF.

Os sintomas da Deficiência Androgênica do Envelhecimento Masculino (DAEM) foram mensurados por meios da Escala dos Sintomas do Envelhecimento Masculino (AMS), versão para o português ${ }^{18}$, composta por 17 questões, com escala de resposta tipo Likert (1 - nenhum; 2 - leve; 3 - moderado; 4 - grave; 5 muito grave) divididas em três blocos de sintomas: psicológicos (cinco questões), somáticos (sete questões) e sexuais (cinco questões). Foram produzidos escores por domínios e geral, baseados no somatório das questões. Os escores variaram de 17 a 85 pontos na escala geral e de 10 a 50 pontos no bloco somático, 3 a 15 pontos bloco psicológico e 4 a 20 pontos bloco sexual. Com base nos valores dos escores os adultos foram classifica- 


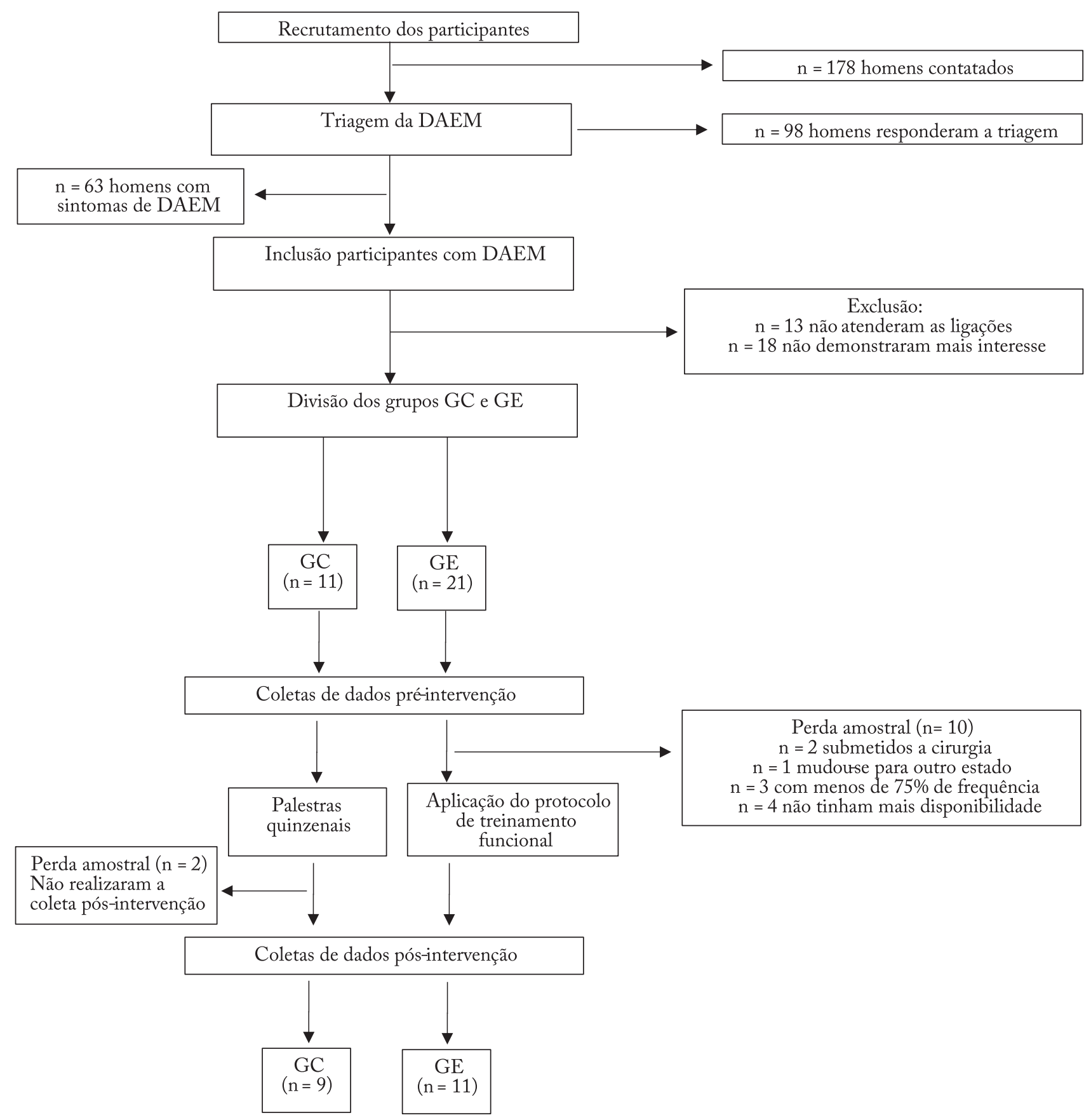

Figura 1 - Fluxograma de seleção amostral e execução da intervenção de treinamento funcional em homens com deficiência androgênica, Florianópolis, Santa Catarina, $2017(\mathrm{n}=20)$.

dos como segue: 27 a 36 pontos. "sintomas leves"; entre 37 a 49 pontos, como "sintomas moderados" e; igual ou superior a 50, como "sintomas graves"19.

Foi utilizado um questionário autoaplicável com questões relacionadas à idade (em anos), escolaridade (fundamental, médio ou superior), estado conjugal (casado ou solteiro), presença de doenças (sim ou não), utilização de medicamentos para doenças e medicamentos para hipertensão, tabagismo, classe econômica.
A classe econômica foi mensurada por meio da renda familiar media mensal, com base nos critérios propostos pelos IBGE (2010), conforme segue: classe A (acima de 20 salário-mínimo); classe B (10 a 20 salário-mínimo); classe $\mathrm{C}$ (4 a 10 salário-mínimo); classe D (2 a 4 salário-mínimo) e classe E (até 2 salário-mínimo), segundo o salário mínimo vigente no ano de 2016 ( $\mathrm{R} \$ 880,00$ reais).

Para avaliação antropométrica foram coletados da- 
dos da massa corporal (MC), estatura (ES) e determinado o índice de massa corporal (IMC). A estatura foi mensurada por meio de uma fita métrica, precisão de $0,1 \mathrm{~cm}$, fixada à parede, com o ponto zero no nível do solo. Para mensurar a massa corporal foi utilizada uma balança digital Plenna Wind MEA 07710. As medidas antropométricas foram realizadas em triplicata e utilizada a de maior valor para fins de resultado final. $\mathrm{O}$ IMC foi obtido pela divisão entre massa corporal $(\mathrm{kg})$ e o quadrado da estatura $\left(\mathrm{m}^{2}\right)$. Para fins de análise, foi categorizado da seguinte forma: peso normal (até 24,9 $\left.\mathrm{kg} / \mathrm{m}^{2}\right)$ e acima do peso $\left(\geq 25 \mathrm{~kg} / \mathrm{m}^{2}\right)^{20}$.

Para mensurar o nível de AF foi utilizado o Questionário Internacional de AF - IPAQ (versão curta) ${ }^{21}$, incluindo as atividades no lazer, deslocamento, doméstico e ocupacional, praticadas por pelo menos $10 \mathrm{mi}^{-}$ nutos. Os adultos informaram se praticaram ou não, a frequência e duração de prática das atividades de intensidade moderada a vigorosa.

Para fins de análise estatística dos dados, com base na frequência e duração da prática de $\mathrm{AF}$, os adultos foram classificados como segue: fisicamente ativo cumpriu com as recomendações de AF vigorosa $\geq 3$ dias/semana e $\geq 20 \mathrm{~min} /$ dia; moderada $\geq 5$ dias/semana e $\geq 30 \mathrm{~min} /$ dia; qualquer atividade somada: $\geq 5$ dias/ semana e $\geq 150 \mathrm{~min} / \mathrm{semana}$; muito ativo: vigorosa $\geq 5$ dias/semana e $\geq 30 \mathrm{~min} /$ dia; vigorosa $\geq 3$ dias/semana e $\geq 20 \mathrm{~min} /$ dia + moderada $\geq 5$ dias $/$ semana e $\geq 30 \mathrm{~min} /$ dia; insuficientemente ativo: não cumpriu com um ou mais desses critérios.

As atividades da intervenção foram desenvolvidas em uma academia de ginástica, localizada na área continental de Florianópolis, por duas pesquisadoras treinadas e uma profissional de Educação Física. Foram realizadas 24 sessões, duas vezes por semana, com duração de 60 minutos cada, em intensidade progressiva. Cada sessão de treino foi composta da forma que segue: 20 minutos de aquecimento, 30 minutos para a atividade principal e 10 minutos para a volta à calma. Como protocolo de intervenção os participantes realizaram treinamento funcional, que aliou atividades aeróbias a exercícios resistidos, de acordo com as recomendações para homens na meia idade ${ }^{22,23}$. As atividades aeróbicas abrangeram a variável agilidade na etapa do aquecimento da aula, e os exercícios resistidos pelas demais variáveis da aptidão física na parte principal da aula (equilíbrio, flexibilidade, resistência, potência, coordenação e força). $\mathrm{O}$ aquecimento e a parte principal incluíram cinco exercícios, executados entre oito e 12 vezes, realizando os circuitos de uma a três vezes, em intensidade progressiva. $\mathrm{O}$ controle $\mathrm{da}$ intensidade do treinamento ocorreu com a presença de músicas de características dinâmicas e enérgicas de 130 a 160 batimento por minuto - bpms (ritmo moderado a intenso). No primeiro mês, os exercícios eram realizados com intensidade leve, no segundo mês com intensidade moderada e nos últimos dois meses com intensidade vigorosa.

A distribuição por frequência (variáveis categóricas) e a média e o desvio padrão (variáveis numéricas) foram utilizadas na análise descritivas das variáveis estudadas. O teste Exato de Fisher foi aplicado para comparar as características gerais (escolaridade, estado conjugal, presença de doenças, utilização de medicamentos para doenças e medicamentos para hipertensão, tabagismo, nível econômico) e nível de $\mathrm{AF}$ entre os grupos GE e GC, no pré e pós-intervenção. A Anova Two Way com medidas repetidas, com post hoc de Sydak, foi utilizado para comparar os valores médios dos minutos em AF de caminhada, moderada, moderada+vigorosa, vigorosa e total intragrupos. Foi testada a interação entre o fator (GE e GC) e o momento (pré e pós-intervenção), por meio da criação de um termo de interação grupo*momento. Adotou-se um nível de significância de 5\%. Todas as análises estatísticas foram realizadas no Pacote Estatístico SPSS - IBM, versão 20.0.

\section{Resultados}

Foram analisados dados completos de 20 adultos do sexo masculino. Na Tabela 1 , estão os resultados referente a caracterização geral dos participantes. Não foram verificadas diferenças significativas entre os grupos para todas as variáveis estudadas no período pré-intervenção. Verificou-se que a maioria tinha ensino superior completo, era de classe média, casado, não era tabagista e 45,5\% dos homens do GE e 88,9\% do GC apresentaram excesso de peso corporal.

Os sintomas da DAEM não apresentaram diferenças significativas entre os $\mathrm{GE}$ e GC nos períodos pré e pós-intervenção. No GE, mesmo não havendo diferença estatisticamente significativa, houve uma diminuição no escore total da DAEM $(38,13 \pm 3,08$ vs 32,11 $\pm 3,14$ pontos; $\mathrm{p}=0,110$ ) e nos sintomas somáticos $(18,04 \pm 1,02$ vs $16,12 \pm 1,07 ; p=0,179)$, psicológicos $(13,05 \pm 1,02$ vs $11,10 \pm 11,09 ; \mathrm{p}=0,207)$ e sexuais $(13,03 \pm$ vs 11,$10 ; p=0,184)$, após a intervenção com treinamento funcional.

A Figura 2 representa o nível de AF dos GE e GC 
Tabela 1 - Características dos homens do Grupo Experimental e do Grupo Controle no período pré-intervenção. Florianópolis, Santa Catarina, $2017(\mathrm{n}=20)$.

\begin{tabular}{lccc}
\hline Variáveis & $\begin{array}{c}\text { Grupo } \\
\text { experimental } \\
\mathrm{n}(\%)\end{array}$ & $\begin{array}{c}\text { Grupo } \\
\text { controle } \\
\mathrm{n}(\%)\end{array}$ & $\mathrm{p}$ valor \\
\hline Escolaridade & & $1(11,1)$ & 0,319 \\
$\quad$ Fundamental & $4(36,4)$ & $1(11,1)$ & \\
$\quad$ Médio & $7(63,6)$ & $7(77,8)$ & \\
$\quad$ Superior & $1(9,1 \%)$ & & 1,000 \\
Classificação econômica & $7(63,6)$ & $6(66,7)$ & \\
Alta (A+B) & $3(27,3)$ & $3(33,3)$ & \\
Média (C) & & & 0,566 \\
$\quad$ Baixa (D/E) & $10(90,9)$ & $7(77,8)$ & \\
Estado conjugal & $1(9,1)$ & $2(22,2)$ & \\
Casado & & & 0,188 \\
Solteiro & $3(27,3)$ & & \\
Índice de massa corporal & $8(72,7)$ & $9(100)$ & \\
Peso normal & & & 0,157 \\
Acima do peso & $6(54,5)$ & $8(88,9)$ & \\
Uso de medicamentos & $5(45,5)$ & $1(11,1)$ & \\
Sim & & & 1,000 \\
Não & $2(18,2)$ & $1(11,1)$ & \\
Tabagista & $9(81,8)$ & $8(88,9)$ & \\
Sim & & & \\
Não & & & \\
\hline
\end{tabular}

Teste Exato de Fisher; $\mathrm{n}$ = frequência absoluta.

no período pré e pós-intervenção. Houve diferença significativa na proporção de adultos classificados como fisicamente ativos no período pós-intervenção entre os grupos. Todos os homens do GE foram classificados como suficientemente ativos, enquanto no GC apenas dois participantes foram classificados da mesma forma $(\mathrm{p}<0,001)$.

Verificou-se um aumento significativo na média de tempo em minutos de prática de caminhada (265

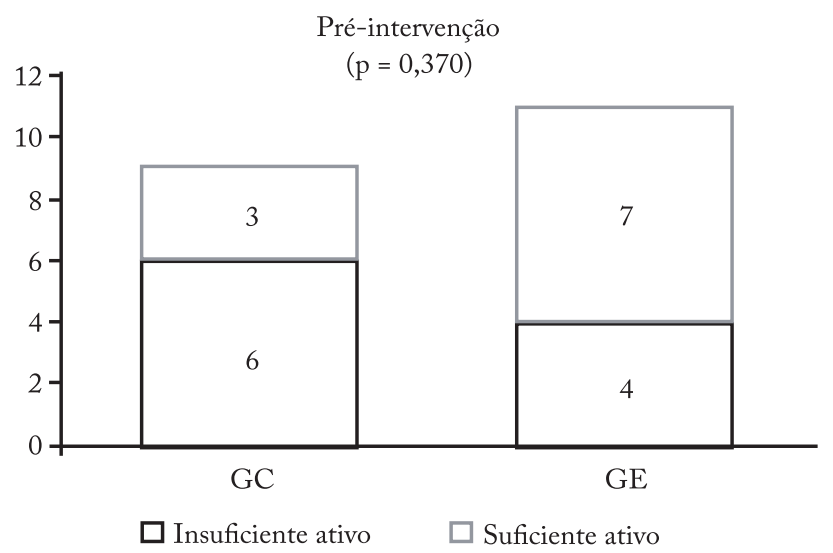

\pm 53 min; $\mathrm{p}=0,013), \mathrm{AF}$ moderada $(138 \pm 21 \mathrm{~min} ; \mathrm{p}=$ $0,004)$, moderada + vigorosa $(209 \pm 24$ min; $p<0,001)$, vigorosa $(71 \pm 48 \mathrm{~min} ; \mathrm{p}<0,011)$ e total $(474 \pm 48 \mathrm{~min}$; $\mathrm{p}<0,001)$ após a intervenção no $\mathrm{GE}$, enquanto que no GC houve diminuição do tempo de prática de AF em todas as intensidades - Tabela 2 .

\section{Discussão}

Foi possível verificar que após a intervenção com treinamento funcional, houve um aumento nos níveis de AF dos homens do GE, bem como no tempo de prática em AF vigorosa, moderada + vigorosa e total. Evidências indicam que a maioria dos homens na meia idade tendem a diminuir o nível de AF, e optar por exercícios de intensidade mais moderada, como a caminhada ${ }^{6,24}$. No presente estudo, a prática do treinamento funcional, e as ações de motivação para a prática de AF favoreceram um aumento significativo nos níveis de $\mathrm{AF}$.

É possível que esse aumento nos níveis de AF também pode ter sido devido a momentos de sociabilização e aos benefícios para a saúde percebidos pelos homens como: melhora nos movimentos corporais do dia a dia, em decorrência do aumento na força muscular promovido pelo treinamento funcional $\mathrm{l}^{25,26} \mathrm{e}$; aumenta da motivação para o envolvimento com atividades fora do programa, como atividades no ambiente doméstico, no lazer e deslocamento ativo, contribuindo assim para o aumento dos níveis de AF dos participantes.

Em relação às intensidades da $\mathrm{AF}$, este estudo indicou que as mais praticadas no período pré-intervenção eram a caminhada no GE e de intensidade moderada+vigorosa no GC. Após o período de intervenção, houve uma diminuição da intensidade das AF praticadas pelo GC e um aumento no $\mathrm{GE}$, sugerindo que a participação na intervenção influenciou no aumento

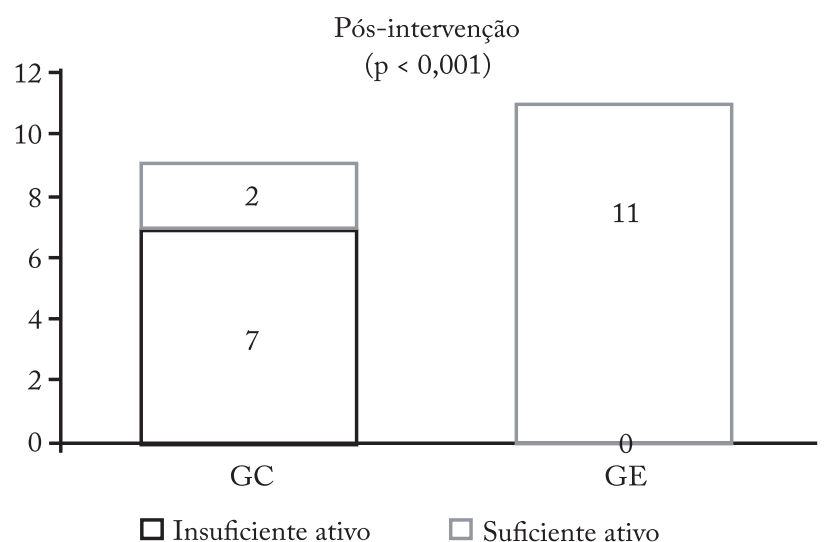

Figura 2 - Nível de AF dos homens do Grupo Experimental e do Grupo Controle no período pré e pós-intervenção. Florianópolis, Santa Catarina, 2017 ( $n=20)$.

Teste Exato de Fisher; Escala de medida = frequência simples. 
Tabela 2 - Comparação do tempo de prática de atividade física entre os grupos experimental e controle nos períodos pré e pós-intervenção com treinamento funcional. Florianópolis, Santa Catarina, $2017(\mathrm{n}=20)$.

\begin{tabular}{|c|c|c|c|c|c|c|c|c|c|}
\hline & \multicolumn{4}{|c|}{$\begin{array}{c}\mathrm{GE} \\
\overline{\mathrm{x}}(\mathrm{dp})\end{array}$} & \multicolumn{4}{|c|}{$\begin{array}{c}\mathrm{GC} \\
\overline{\mathrm{x}}(\mathrm{dp})\end{array}$} & \multirow[b]{2}{*}{$\mathrm{p}$-valor\# } \\
\hline & Pré & Pós & p-valor* & $\mathrm{ME}$ & Pré & Pós & $\mathrm{p}$ valor** & $\mathrm{ME}$ & \\
\hline \multicolumn{10}{|l|}{ Atividade Física } \\
\hline Caminhada (min/sem) & $158(42)$ & $265(53)$ & 0,154 & 106 & $54(46)$ & $48(59)$ & 0,934 & -7 & 0,013 \\
\hline Moderada (min/sem) & $80(29)$ & $138(21)$ & 0,070 & 58 & $86(33)$ & $34(23)$ & 0,140 & -51 & 0,004 \\
\hline Vigorosa (min/sem) & $8(13)$ & $71(18)$ & 0,011 & 63 & $20(14)$ & $18(20)$ & 0,947 & -2 & 0,071 \\
\hline Moderada+vigorosa $(\mathrm{min} / \mathrm{sem})$ & $89(31)$ & $209(24)$ & 0,011 & 120 & $106(34)$ & $53(26)$ & 0,276 & -53 & $<0,001$ \\
\hline Total (min/sem) & $247(40)$ & $474(48)$ & 0,002 & 227 & $160(44)$ & $101(53)$ & 0,393 & -59 & $<0,001$ \\
\hline
\end{tabular}

GE = Grupo Experimental $(\mathrm{n}=11) ; \mathrm{GC}=$ Grupo Controle $(\mathrm{n}=9) ; \overline{\mathrm{x}}=$ média; $\mathrm{dp}=$ desvio padrão; $\mathrm{ME}=$ mudança de escore; ${ }^{*} \mathrm{p}$ valor para comparação entre os períodos pré e pós do GE; ** p valor para comparação entre os períodos pré e pós do G; \# p valor para comparação entre GE e GC no período pós-intervenção; Teste ANOVA Two-Way com medidas repetidas e teste de comparação de Sydak.

das intensidades de AF praticadas. Conforme relatos espontâneos dos participantes, além da prática do treinamento funcional, o incentivo por parte das pesquisadoras para a prática de $\mathrm{AF}$ também exerceu influência para que essa alteração ocorresse. Esse é um achado importante do ponto de vista de saúde pública, tendo em vista que o aumento da prática de $\mathrm{AF}$ diária na meia idade pode contribuir para um efeito positivo sobre as doenças cardiovasculares e seus fatores de risco ${ }^{24}$ e nas síndromes metabólicas ${ }^{27 .}$

Presume-se que o treinamento funcional é um exercício benéfico para homens com DAEM, que além de incentivar e motivar a prática de $\mathrm{AF}$ nos diferentes domínios e contextos, também pode ser considerada benéfica para a saúde geral, por ser composto de exercícios aeróbios e resistidos, os quais contribuem para o aumento nos níveis de força muscular, diminuição dor nas articulações, dores musculares, insônia, e favorecem a sensação de bem-estar geral. Em conjunto, esses fatores melhoram os sintomas da DAEM ${ }^{9}$.

Um estudo com homens de meia idade, identificou melhorias significativas na qualidade de vida após um ano de intervenção com AF de intensidade moderada a vigorosa ${ }^{28}$. Além de o exercício físico e a AF atuarem de uma maneira positiva no controle dos aspectos psicológicos, somáticos e sexuais, presentes nos sintomas da DAEM $^{29}$. Esses achados, reforçam a importância e a necessidade de implementação de programas de AF para aumentar o nível e a intensidade da AF nos homens de meia idade com DAEM.

É importante salientar que este estudo apresentou algumas limitações, como o fato da amostra não ter sido randomizada, devido à dificuldade de os homens com os sintomas da DAEM e a aceitação em fazer parte do estudo. Esta opção pode ter produzido um viés de sele- ção. É possível que aqueles que optaram por participar da intervenção poderiam possuir previamente, maior expectativa e pré-disposição à melhores resultados do que aqueles que não optaram. Assim, os resultados obtidos podem ser associados não apenas à intervenção, mas também a escolha dos homens pelos grupos. No entanto, observou-se que em ambos os grupos não foi identificada diferenças significativas no período pré-intervenção em nenhuma das variáveis estudadas. O uso de questionário para mensurar o nível de $\mathrm{AF}$ pode ter superestimado o tempo de prática. $\mathrm{O}$ fato de se ter sido utilizado o IPAQ - versão curta pode ser outro fator limitante, uma vez que diferentemente da versão longa, a curto não discrimina em quais domínios (ocupacional, deslocamento, lazer e ambiente doméstico) a atividade foi praticada.

Por outro lado, destaca-se que este foi possivelmente um dos primeiros estudos a investigar a influência de intervenção com AF em homens com DAEM, lembrando que se trata de uma população difícil de ser atingida pelo preconceito aos cuidados com a saúde e o não conhecimento da DAEM.

Todavia, apesar dessa dificuldade, foi possível observar um envolvimento dos participantes no programa de intervenção que influenciou nos achados do presente estudo, aumentando o nível de AF dos homens. Assim, foi possível identificar neste estudo, que os homens que participaram das aulas de treinamento funcional apresentaram um aumento nos níveis de atividade física, bem como no tempo na intensidade praticada, em relação ao período pré-intervenção. Dessa forma, um programa de intervenção com treinamento funcional, pode ser um fator motivador para a incorporação da $\mathrm{AF}$ em diferentes contextos no cotidiano dos participantes. 


\section{Conflito de interesses}

Os autores declaram não haver conflito de interesses.

\section{Contribuição dos autores}

Wolfgramm BR, participou desde a escolha da temática, submissão ao comitê de ética, coleta de dados, análise estatística, descrição dos resultados, discussão de dados e conclusão; Vieira MCS, participou da coleta de dados, redação, revisão do artigo e aprovação final da versão a ser publicada; Guimarães ACAG, participou da redação do artigo, conceito intelectual do artigo, redação e revisão do artigo.

\section{Referências}

1. Guallar-Castillón $P$, Bayán-Bravo $A$, León-Muñoz LM, Balboa-Castillo T, López-García E, Gutierrez-Fisac JL, et al. The association of major patterns of physical activity, sedentary behavior and sleep with health-related quality of life: a cohort study. Prev Med. 2014;67:248-54.

2. Brasil. Ministério da saúde. Brasília. Aumento na prática de atividades físicas, 2014 [acesso em 2017 jan 26]. Disponível em: http://www.brasil.gov.br.

3. Del Duca GF, Nahas MV, Hallal PC, Peres KG. Atividades físicas no lazer entre adultos de Florianópolis, Santa Catarina, Brasil: estudo populacional sobre as características das práticas e de seus praticantes. Cien Saude Colet. 2014;19(11):4595-04.

4. Sattelmair J, Pertman J, Ding EL, Kohl HW, 3rd, Haskell W, Lee IM. Dose response between physical activity and risk of coronary heart disease: a meta-analysis. Circulation. 2011;124(7):789-95.

5. Department of Health and Ageing. National male health policy: Building on the strengths of Australian males. Canberra: Department of Health and Ageing; 2010.

6. Mabry RM, Winkler EA, Reeves MM, Eakin EG, Owen N. Correlates of Omani adults' physical inactivity and sitting time. Public Health Nutr. 2013;15(11):65-72.

7. Antunes PC, Silva AM. Elementos sobre a concepção de meia idade, no processo de envelhecimento humano. Rev Kairós Geront. 2013;19(5):123-40.

8. Millar AC, Lau ANC, Tomlinson G, Kraguljac A, Simel DL, Detsky AS, et al. Predicting low testosterone in aging men: a systematic review. Can Med Assoc J. 2016;188(13):321-30.

9. Heinemann LA. Aging Males' Symptoms scale: a standardized instrument for the practice. J Endocrinol Invest. 2005;28(11):34-38.

10. Rohden F. A "criação" da andropausa no Brasil: articulações entre ciência, mídia e mercado e redefinições de sexualidade e envelhecimento. Psic Cono Soc. 2012;2(2):196-219.

11. Vieira MCS, Cardoso AA, Guimarães ACA. Male aging symptoms: the positive influence of moderate and total physical activity. Rev Bras Cineantopom Desempenho Hum. 2016;18(4):460-70.

12. Condorelli RA, Di Mauro M, Mongioì LM, Russo GI, Morgia G, La Vignera S. Effects of tadalafil treatment combined with physical activity in patients with low onset hypogonadism: results from a not-randomized single arm phase 2 study. Aging Male. 2016;19(3):155-60.
13. Pelletier-Beaumont E, Arsenault BJ, Alméras N, Bergeron J, Tremblay A, et al. Normalization of visceral adiposity is required to normalize plasma apolipoprotein B levels in response to a healthy eating/physical activity lifestyle modification program in viscerally obese men. Atherosclerosis. 2012;221(2):577-82.

14. American College of Sports Medicine. ACSM's Guidelines for Exercise Testing and Prescription. 9th ed. Philadelphia, Pa: Lippincott Williams and Wilkins; 2014.

15. Heinrich KM, Becker C, Carlisle T, Gilmore K, Hauser J, Frye J, et al. High-intensity functional training improves functional movement and body composition among cancer survivors: a pilot study. Eur J Cancer Care. 2015;24:812-17.

16. Román PAL, Campos MAS, García-Pinillos F. Effects of functional training on pain, leg strength and balance in women with fibromyalgia. Mod Rheumatol. 2015;25:943-47.

17. Barbosa MPCR, Junior JM, Cassemiro BM, Bernardo AFB, Silva AKF, Vanderlei FM, et al. Effects of functional training on geometric indices of heart rate variability. J Sport Health Sci. 2016;5:183-9.

18. Heinemann LA, Saad F, Zimmermann T, Novak A, Myon E, Badia X, et al. The Aging Males'Symptoms (AMS) scale: update and compilation of international versions. Health Qual Life Outcomes. 2003;1(1):34-8.

19. Heinemann LAJ, Zimmermann T, Vermeulen A, Thiel C, Hummel W. A new Aging Male's Symptoms' (AMS) rating scale. Aging Male. 1999;2:105-14.

20. Health Organization. In: Global recommendations on physical activity for health, 18-64 years old. 2011. Acesso em: http://www.who.int/dietphysicalactivity/physical-activityrecommendations-18-64years.pdf. [Out 2016].

21. Pardini R, Matsudo S, Matsudo V, Araújo T, Andrade E, Braggion $\mathrm{G}$, et al. Validation of international physical activity questionnaire (IPAQ): pilot study in brazilian young adults. Med Scienc Sports Exerc. 1997;29(6):S5-S9.

22. Condorelli RA, Calogero AE, Di Mauro M, Mongiò̀ LM, Russo GI, Morgia G, et al. Effects of tadalafil treatment combined with physical activity in patients with low onset hypogonadism: results from a not-randomized single arm phase 2 study. Aging Male. 2016;6:1-6.

23. Shen S, Lu Y, Dang Y, Qi H, Shen Z, Wu L, et al. Effect of aerobic exercise on the atherogenic index of plasma in middle-aged Chinese men with various body weights. Int J Cardiol. 2016.230:1-5.

24. Mour BP, Marins JCB, Amorim PRS. Self selected walking speed in overweight adults: Is this intensity enough to promote health benefits? Apunts Med Esport. 2011;46(169):11-5.

25. Rocha AS, Marega M. Impacto da intervenção motivacional no aumento do nível de atividade física. Eintein. 2010;8(1 Pt 1):46-52.

26. Godfrey A, Lord S, Galna B, Mathers JC, Burn DJ, Rochester $\mathrm{L}$. The association between retirement and age on physical activity in older adults. Age Ageing. 2014;43(3):386:93.

27. WHO. World Health Organization 2017. Physical Activity. 2017. Acesso em: <http://www.who.int/mediacentre/ factsheets/fs385/en/>. [2017 Junho].

28. Atashak S, Stannard SR, Azizbeigi K. Cardiovascular risk factors adaptation to concurrent training in overweight sedentary middle-aged men. J Sports Med Phy Fitness. 2016;56(5):624-30. 
29. Marie-Ludivine CD, Papouin G, Saint-Val P, Lopez A. Effect of adapted karate training on quality of life and body balance in 50-year-old men. Open Access J Sports Med. 2010;4(1):143-50.

Recebido: $11 / 12 / 2017$

Aprovado: 28/07/2018

\section{Como citar este artigo:}

Wolfgramm BR, Vieira MCS, Guimarães ACA. Treinamento funcional em homens com deficiência androgênica do envelhecimento masculino. Rev Bras Ati Fis Saúde. 2018;23:e0040. DOI: 10.12820/rbafs.23e0040 